\title{
Risk for Colorectal Adenomas Among Patients with Pancreatic Intraductal Papillary Mucinous Neoplasms: a Prospective Case- Control Study
}

\author{
Nikola Panic ${ }^{1,2}$, Gabriele Capurso ${ }^{3}$, Fabia Attili ${ }^{1}$, Giovanna Vitale ${ }^{1}$, Serena Stigliano ${ }^{3}$, Gianfranco Delle Fave ${ }^{3}$, \\ Emanuele Leoncini $^{2}$, Milutin Bulajic ${ }^{1}$, Emilio Di Giulio ${ }^{3}$, Guido Costamagna ${ }^{1}$, Stefania Boccia ${ }^{2}$, Alberto Larghi ${ }^{1}$
}

1) Digestive Endoscopy Unit;

2) Institute of Public Health,

Section of Hygiene, Catholic University;

3) Digestive and Liver Disease Unit, S. Andrea Hospital, Sapienza University of Rome Rome, Italy

\footnotetext{
Address for correspondence: Alberto Larghi MD, $\mathrm{PhD}$ Digestive Endoscopy Unit, Catholic University Largo A. Gemelli 8, 00168 Rome, Italy albertolarghi@yahoo.it
}

Received: 30.08.2015 Accepted: 14.10.2015

\begin{abstract}
Background \& Aims: It has been reported that patients with intraductal papillary mucinous neoplasms of the pancreas are at an increased risk of colorectal cancer. The aim of our study was to investigate whether patients with intraductal papillary mucinous neoplasms are at a higher risk of colorectal adenomas with respect to the general population, as this condition represents the precursor of sporadic colorectal cancer.

Methods: A case-control study was conducted at the Catholic University and University Sapienza, Rome, Italy. The cases were patients with intraductal papillary mucinous neoplasms without history of colorectal cancer, who had underwent screening colonoscopy for the first time. The controls were individuals who had underwent first time colonoscopy for screening or evaluation of non-specific abdominal symptoms. Chi-square and Fisher tests were used to compare the distributions of categorical variables.

Results: We enrolled 122 cases and 246 controls. Colorectal polyps were found in 52 cases (42.6\%) and 79 controls $(32.1 \%)(p<0.05)$. In 29 cases $(23.8 \%)$ and 57 controls $(23.2 \%)$ histological examination disclosed adenomatous polyps $(\mathrm{p}=0.90$ ). There was no difference between the groups in relation to the presence of polyps with low-grade ( $19.7 \%$ vs. $19.8 \%, \mathrm{p}=0.98$ ) and high-grade dysplasia ( $4.9 \%$ vs. $4.5 \%$, $\mathrm{p}=0.85$ ).

Conclusion: Patients with intraductal papillary mucinous neoplasms of the pancreas are not at an increased risk for the development of adenomatous colorectal polyps.
\end{abstract}

Key words: IPMN - colorectal cancer - colonoscopy - adenomatous polyps.

Abbreviations: BD-IPMN: branch duct intraductal papillary mucinous neoplasm; CRC: colorectal cancer; FAP: familiar adenomatous polyposis; FNA: fine needle aspiration; FOBT: fecal occult blood test; HNPCC: hereditary non-polyposis colorectal cancer; IPMN: intraductal papillary mucinous neoplasm; M-IPMN: mixed intraductal papillary mucinous neoplasm; MD-IPMNs: main duct intraductal papillary mucinous neoplasm; PDAC: pancreatic ductal adenocarcinoma; S-MRCP: magnetic resonance cholangiopancreatography with secretin stimulation.

\section{INTRODUCTION}

Intraductal papillary mucinous neoplasms (IPMNs) of the pancreas have been previously reported to be associated with an increased risk of developing extra-pancreatic tumours [113]. Though some authors have reported an increased risk for gastric, lung, breast, kidney and thyroid cancers [13], the most frequent extra-pancreatic tumour consistently found in these patients has been colorectal cancer (CRC) $[3,6,8,9,11,13]$.
Colorectal cancer represents the third most common cancer worldwide with more than 1.3 million new cases (9.7\% of total) and almost 700,000 deaths every year [14]. Almost $55 \%$ of the cases occur in more developed regions [14], where the prognosis is relatively favourable with a 5-year survival rate reaching 65\% in USA, Canada, Australia and several European countries $[15,16]$. On the other hand, the incidence is increasing in countries or areas with poor healthcare resources [17], where a 5-year survival is no more than $50 \%$ [18]. Identification of all risk factors associated with CRC becomes of paramount importance to apply properly designed screening programs to those at higher risk than the general population in order to detect precancerous lesions or cancers at an early and more curable stage.

The mechanism(s) behind the association between IPMN and CRC is still unknown. It is well known that almost all 
sporadic CRCs develop slowly over several years through the adenoma-carcinoma carcinogenetic sequence [19]. Thus, it is possible to hypothesize that the increased risk of colorectal CRC in IPMN patients may be related to an increased propensity to develop colorectal adenomas, which represent the precursors of sporadic CRC. Some authors have previously reported an increased prevalence of colorectal polyps in patients with IPMN, based on which they proposed to consider screening colonoscopy for all patients with IPMN [8]. This inference, however, has been based only on the result of this single study that was retrospective and based on a chart review, while no data coming from studies specifically designed to prospectively assess the rate of colonic polyps in IPMN patients are available.

To answer this important question, we conducted a twocentre case-control study aimed at evaluating the prevalence of colorectal adenomas in consecutively enrolled Italian patients with IPMN undergoing first time screening colonoscopy as compared to matched controls.

\section{MATERIAL AND METHODS}

A two centre prospective case-control study was conducted at the Digestive Endoscopy Unit of the Catholic University and the Digestive and Liver Disease Unit of the University Sapienza, Rome, Italy between January 2012 and December 2013. Detailed methodology has been previously described [13, 20]. Cases were prevalent IPMN who had underwent screening colonoscopy for the first time in their life. The criteria for the IPMN diagnosis were previously described [21]. The diagnosis of IPMN was considered as certain in the presence of either histological diagnosis obtained by endoscopic ultrasound (EUS) or surgical specimen, or cytologic diagnosis obtained by EUS. A highly probable diagnosis of IPMN was based on the presence of one or several main pancreatic duct and/or branch duct dilatation(s) and/or pancreatic cystic lesions communicating with pancreatic ducts at computed tomography (CT), magnetic resonance cholangiopancreatography with secretin stimulation (S-MRCP), endoscopic retrograde cholangiopancreatography (ERCP) or EUS. Patients were excluded if they had a history of CRC, if they had undergone a previous colonoscopy independently on the presence or absence of CRC and/ or colonic polyps, and if they had cystic lesions other than IPMN. Controls matched to each IPMN case (2:1) by gender and age ( \pm 5 years) were enrolled alongside among individuals who had underwent their first colonoscopy for screening or for evaluation of non-specific abdominal symptoms at both institutions. Individuals who underwent colonoscopy because of a personal history of CRC, of familiar adenomatous polyposis (FAP) or hereditary non-polyposis colorectal cancer (HNPCC), positivity to fecal occult blood test (FOBT), iron deficiency anaemia and bright red blood per rectum were excluded. Both cases and controls were interviewed by trained physicians using a structured questionnaire and data on demographics, lifestyle habits (alcohol consumption, cigarette smoking), prior medical history, cancer family history and medication use were collected.

Cases and controls underwent screening colonoscopy and the prevalence of colon polyps and/or CRC was determined. Polyethylene glycol lavage solution was used for colon preparation. Colonoscopies were conducted by experienced endoscopists from the Digestive Endoscopy Unit of the Catholic University (A.L., F.A.) and the Digestive and Liver Disease Unit of the University Sapienza (E.D.G.) Anatomical landmarks (Bauhin valve and appendix orifice) were recognized as proof that the entire colon had been examined. Patients who were diagnosed with CRC were referred to surgical evaluation, while all detected polyps were removed whenever possible. A histopathologic examination of all removed polyps was performed and histologic type of the polyp and the degree of dysplasia were determined. All colonoscopies were performed under conscious sedation (midazolam and phentanyl e.v.) and patients were observed in the recovery unit for 1 hour after the procedure. For those with positive colonoscopy findings, surveillance was suggested after proper treatment and histopathologic evaluation, according to current guidelines [22].

For IPMN cases data on IPMN characteristics, such as type of ductal involvement, focality, maximal dilation of the duct and presence of nodules or solid tissue inside the cyst cavity were collected. The type of duct involvement was determined by the revision of clinical imaging studies and/or based on endoscopic ultrasound (EUS) evaluation, and classified as either main duct (MD-IPMNs), branch duct (BD-IPMNs) or mixed (M-IPMNs).

We conducted a descriptive analysis using relative frequencies and percentages to summarize the characteristics of the IPMN patients at the time of diagnosis. Chi-square and Fisher exact tests were used to compare the distribution of categorical variables between patients with IPMNs and control subjects. Additionally, as we expected that the prevalence of colorectal polyps might differ between the compared groups based on the family history of CRC, we planned a priori to stratify our data according to the 1st-degree family history of CRC. Statistical analyses were performed using Stata software (StataCorp. 2013. Stata Statistical Software: Release 13. College Station, TX: StataCorp LP).

\section{RESULTS}

One hundred and twenty-two patients with IPMN and 244 matched controls were prospectively enrolled in the study. Demography of the cases and characteristics of the IPMN lesions are reported in Table I. Patients with IPMN were predominantly female $(61.5 \%)$, with an age over 60 years in $69.6 \%$ of the cases. The large majority of the cases were branch duct (BD)-IPMN (87.7\%) multifocal (67.2\%) and without evidence of intra-lesional nodules $(81.1 \%)$. The mean diameter of the largest lesions was $16.7 \pm 7.9 \mathrm{~mm}$, while the mean dilatation of the main pancreatic duct in main duct (MD)IPMN was $9.0 \pm 5.26 \mathrm{~mm}$ (Table I). EUS \pm FNA was performed in $80 \%$ of the IPMNs and overall they were treated conservatively with clinical follow up in $86.9 \%$ of the cases (Table I).

Table II reports demographics, lifestyle habits, previous medical history and cancer family history of IPMN cases and controls (Table II). Cases were significantly more likely to be drinkers $(\mathrm{p}<0.01)$ or to drink $\geq 21$ drinks per week $(\mathrm{p}<0.05)$. They also had significantly more frequently a history of chronic pancreatitis $(p<0.001)$, diabetes mellitus $(p<0.001)$, 
Table I. Clinical features of the 122 patients with intraductal papillary mucinous neoplasms of the pancreas at the time of diagnosis

\begin{tabular}{|c|c|c|}
\hline & Number & $\%$ \\
\hline \multicolumn{3}{|l|}{ Gender } \\
\hline Male & 47 & $38.5 \%$ \\
\hline Female & 75 & $61.5 \%$ \\
\hline \multicolumn{3}{|l|}{ Age (years) } \\
\hline$<50$ & 15 & $12.3 \%$ \\
\hline $50-59$ & 22 & $18.0 \%$ \\
\hline $60-69$ & 43 & $35.2 \%$ \\
\hline$\geq 70$ & 42 & $34.4 \%$ \\
\hline \multicolumn{3}{|l|}{ Duct involvement } \\
\hline BD-IPMN & 107 & $87.7 \%$ \\
\hline MD-IPMN & 7 & $5.7 \%$ \\
\hline C-IPMN & 8 & $6.6 \%$ \\
\hline \multicolumn{3}{|l|}{ Focality } \\
\hline Unifocal & 40 & $32.8 \%$ \\
\hline Multifocal & 82 & $67.2 \%$ \\
\hline Branch duct maximum dilatation $(\mathrm{mm})(\S)$ & 16.69 & $12.0-21.0$ \\
\hline Wirsung maximal dilatation $(\mathrm{mm})(\S)$ & 9.0 & $5.0-10$ \\
\hline \multicolumn{3}{|l|}{ Nodules } \\
\hline No & 99 & $81.1 \%$ \\
\hline Yes & 23 & $18.9 \%$ \\
\hline \multicolumn{3}{|l|}{ Surgery } \\
\hline No & 106 & $86.9 \%$ \\
\hline Yes & 16 & $13.1 \%$ \\
\hline \multicolumn{3}{|l|}{ Endosonography } \\
\hline No & 27 & $22.1 \%$ \\
\hline Yes & 95 & $77.9 \%$ \\
\hline
\end{tabular}

(\$) Median and interquartile range; IPMN, intraductal papillary mucinous neoplasm; $\mathrm{BD}$, branch duct; $\mathrm{MD}$, main duct; $\mathrm{C}$, combined

both insulin-dependent $(\mathrm{p}<0.001)$ and non-insulin dependent $(\mathrm{p}<0.05)$, as well as a previous history of cholecystectomy $(\mathrm{p}<0.01)$ (Table II). With regard to cancer family history, IPMN patients were significantly more likely to have 2nddegree family history for any cancer $(\mathrm{p}<0.05)$, and 2nd-degree family history for pancreatic ductal adenocarcinoma (PDAC) $(\mathrm{p}<0.05)$. On the other hand, controls were significantly more likely to have a 1st-degree relative with a history of CRC $(\mathrm{p}<0.001)$.

In all cases and controls the entire colon was examined. Colorectal polyps were found in 52 IPMNs (42.6\%) and in 79 controls $(32.1 \%)(\mathrm{p}<0.05)$ (Table II). Mean polyp diameter was $6.1 \pm 5.28 \mathrm{~mm}$. In 29 cases $(23.8 \%)$ and 57 controls $(23.2 \%)$ histologic examination disclosed adenomatous polyps, which were multiple in 11 cases (9\%) and 20 controls (8.1\%) (Table II). There was no difference between the groups also in regard to presence of polyps with low-grade dysplasia or high-grade dysplasia (Table II). Three cases of CRC were detected, 2 in IPMNs (1.6\%) and 1 in the controls (0.4\%), but the frequency was too small to make any comparison between the groups.

As we expected that 1st-degree family history of CRC might affect the prevalence of colorectal polyps, Table III reports the data stratified according to this covariate. No difference in the prevalence of colorectal polyps or adenomatous polyps with low-grade or high-grade dysplasia between IPMN cases and controls among those with 1st-degree family history of CRC was observed. A slightly higher prevalence of colorectal polyps among cases than controls without 1st-degree family history of CRC was reported ( $43.3 \%$ vs. $31.4 \%, \mathrm{p}=0.051$ ) (Table III). However, when restricting the analysis to adenomatous polyps, no difference between IPMN cases and controls without 1stdegree family history of CRC was observed (Table III).

\section{DISCUSSION}

We conducted a case-control study in order to evaluate the prevalence of colorectal adenomas in prospectively enrolled Italian patients with IPMN undergoing first time screening colonoscopy. Compared to a matched control population of individuals who underwent colonoscopy for screening or for evaluation of non-specific abdominal symptoms, no increased prevalence of adenomatous polyps was found in IPMN patients. This result did not show an increased propensity to develop colorectal adenomas among IPMN patients.

Colorectal carcinoma has been the most frequent extrapancreatic tumour consistently found in IPMN patients $[3,6,13]$. Eguchi et al. [3] reported that IPMN is a strong independent risk factor for preoperative CRC. They found that CRC occurred 5.37 times more frequently in IPMN patients than in the general population [3]. Rial et al. [6] reported 1.66 times higher rate of CRC in patients with invasive IPMN as compared to US general population and our study group previously reported that IPMN patients in Italy harbour CRC 2.26 times more frequently than expected [13]. The mechanism(s) for the association between IPMN and CRC has not been elucidated so far. One important point has been raised by Reid-Lombardo et al. [8], who performed a large case-control retrospective study at the Mayo Clinic. They found adenomatous colorectal polyps to be present 2 and 1.4 times more frequently in patients with IPMN as compared to patients with pancreatic adenocarcinoma and to the general population, respectively. These findings prompted the authors to advocate screening colonoscopy for all patients with IPMN.

The adenoma-carcinoma sequence is a well-known carcinogenetic mechanism that is responsible for almost all sporadic CRCs [19]. Colorectal cancer develops from the progressive transformation of adenomatous polyps [19] through a series of molecular events including APC gene mutation [23], as well as KRAS and TP53 inactivation [24]. Based on this very well-known mechanism and the results of the study by Reid-Lombardo et al. [8], we performed a study to test the hypothesis that the risk of CRC development in patients with IPMN could be related to an increased propensity to harbour colorectal adenomas.

Contrary to the study by Reid-Lombardo et al. [8] that was based on a retrospective chart review with possible bias toward an increased prevalence of adenomatous polyps in cases because of an increased probability of undergoing diagnostic tests including colonoscopy, we evaluated the prevalence of colorectal polyps among a large cohort of consecutively enrolled patients with IPMN undergoing first time colonoscopy. As compared with a matched control population also undergoing 
Table II. Characteristics of patients with intraductal papillary mucinous neoplasms of the pancreas and controls by selected variables of family history chronic conditions, medication and lifestyles, and polyp findings according to colonoscopy

\begin{tabular}{|c|c|c|c|c|c|}
\hline \multirow[b]{2}{*}{ Male sex } & \multicolumn{2}{|c|}{ Cases $(n=122)$} & \multicolumn{2}{|c|}{ Controls $(n=246)$} & \multirow{2}{*}{$\begin{array}{c}\mathrm{p} \text { value } \\
0.95\end{array}$} \\
\hline & 47 & $38.5 \%$ & 94 & $38.2 \%$ & \\
\hline Age & 63,4 & \pm 11.3 & 62,3 & \pm 10.9 & 0.25 \\
\hline \multicolumn{6}{|l|}{ Cigarette smoking } \\
\hline Ever & 64 & $53.8 \%$ & 115 & $46.7 \%$ & 0.21 \\
\hline$\geq 20$ pack-years & 33 & $28.2 \%$ & 48 & $19.5 \%$ & 0.06 \\
\hline \multicolumn{6}{|l|}{ Alcohol } \\
\hline Ever & 53 & $44.5 \%$ & 75 & $30.5 \%$ & $<0.01$ \\
\hline$\geq 21$ drinks per week & 10 & $9.0 \%$ & 7 & $2.9 \%$ & $<0.05$ \\
\hline \multicolumn{6}{|l|}{ Medical history } \\
\hline History of chronic pancreatitis & 8 & $6.7 \%$ & 0 & $0.0 \%$ & $<0.001$ \\
\hline History of diabetes & 23 & $19.3 \%$ & 18 & $7.3 \%$ & $<0.001$ \\
\hline History of insulin-dependent diabetes & 6 & $5.0 \%$ & 0 & $0.0 \%$ & $<0.001$ \\
\hline History of noninsulin-dependent diabetes & 17 & $14.3 \%$ & 16 & $6.7 \%$ & $<0.05$ \\
\hline History of peptic ulcer & 4 & $3.3 \%$ & 3 & $1.2 \%$ & 0.23 \\
\hline History of cholecystectomy & 20 & $16.7 \%$ & 17 & $6.9 \%$ & $<0.01$ \\
\hline \multicolumn{6}{|l|}{ Cancer family history } \\
\hline Any cancer (1st degree) & 70 & $57.9 \%$ & 134 & $57.8 \%$ & 0.99 \\
\hline Any cancer (2nd degree) & 22 & $18.2 \%$ & 24 & $10.3 \%$ & $<0.05$ \\
\hline PDAC (1st degree) & 12 & $9.9 \%$ & 13 & $5.3 \%$ & 0.10 \\
\hline PDAC (2nd degree) & 4 & $3.3 \%$ & 0 & $0.0 \%$ & $<0.05$ \\
\hline \multicolumn{6}{|l|}{ Common sites, first-degree family history } \\
\hline Colorectal cancer & 17 & $14.1 \%$ & 93 & $37.8 \%$ & $<0.001$ \\
\hline Gastric cancer & 9 & $7.4 \%$ & 12 & $4.9 \%$ & 0.32 \\
\hline Breast cancer & 6 & $5.0 \%$ & 21 & $8.5 \%$ & 0.22 \\
\hline Lung cancer & 12 & $9.9 \%$ & 18 & $7.3 \%$ & 0.39 \\
\hline Uterine cancer & 3 & $2.5 \%$ & 4 & $1.6 \%$ & 0.69 \\
\hline Melanoma & 3 & $2.5 \%$ & 3 & $1.2 \%$ & 0.40 \\
\hline Hepatocellular carcinoma & 6 & $5.0 \%$ & 5 & $2.0 \%$ & 0.19 \\
\hline \multicolumn{6}{|l|}{ Use of drugs } \\
\hline Aspirin & 16 & $13.2 \%$ & 34 & $14.3 \%$ & 0.78 \\
\hline Statins & 20 & $16.5 \%$ & 49 & $20.6 \%$ & 0.36 \\
\hline Insulin & 7 & $5.8 \%$ & 0 & $0.0 \%$ & $<0.001$ \\
\hline Colon polyps & 52 & $42.6 \%$ & 79 & $32.1 \%$ & $<0.05$ \\
\hline Adenomatous polyps & 29 & $23.8 \%$ & 57 & $23.2 \%$ & 0.9 \\
\hline Multiple adenomatous polyps & 11 & $9.0 \%$ & 20 & $8.1 \%$ & 0.77 \\
\hline Low grade dysplasia & 24 & $19.7 \%$ & 48 & $19.5 \%$ & 0.98 \\
\hline High grade dysplasia & 6 & $4.9 \%$ & 11 & $4.5 \%$ & 0.85 \\
\hline
\end{tabular}

Data are mean (SD) for continuous variables or number of individuals (\%) for discrete variables; PDAC, pancreatic ductal adenocarcinoma

first time colonoscopy for screening purposes or to evaluate non-specific symptoms, we found a higher prevalence of colorectal polyps among IPMN patients. When considering adenomatous polyps only, however, this difference was no longer significant. Furthermore, these results were confirmed after stratifying according to the 1st-degree family history of CRC, which might affect the presence of colorectal adenomas. Therefore our study did not confirm the findings of ReidLombardo et al. [8]. However, when interpreting our results, it should be kept in mind that the above mentioned study included more MD-IPMNs and C-IPMNs. It could be a case that patients harbouring these more invasive IPMN types are more prone to develop colorectal adenomas.

Overall, the results of our study suggest that factors other than an increased propensity of IPMN patients to harbour colorectal adenomas could be responsible for the increased occurrence of CRC in these patients. It could be hypothesised that the mechanism for an increased risk of CRC in IPMN 
Table III. Prevalence of colon polyps in intraductal papillary mucinous neoplasms of the pancreas cases and controls according to family history of colorectal cancer

\begin{tabular}{lccccc}
\hline 1st-degree family history of CRC & \multicolumn{4}{l}{ Cases $(\mathrm{n}=104)$} & \multicolumn{2}{c}{ Controls $(\mathrm{n}=153)$} & $\mathrm{p}$-value \\
\hline Colon polyps & 45 & $43.4 \%$ & 48 & $31.4 \%$ & 0.051 \\
Adenomatous polyps & 26 & $25.0 \%$ & 36 & $23.5 \%$ & 0.79 \\
Multiple adenomatous polyps & 10 & $9.6 \%$ & 13 & $8.5 \%$ & 0.76 \\
Low grade dysplasia & 21 & $20.2 \%$ & 30 & $19.6 \%$ & 0.90 \\
High grade dysplasia & 8 & $7.7 \%$ & 8 & $5.2 \%$ & 0.88 \\
\hline No 1st-degree family history of CRC & Cases $(\mathrm{n}=17)$ & Controls $(\mathrm{n}=93)$ & $\mathrm{p}$-value \\
\hline Colon polyps & 6 & $35.3 \%$ & 31 & $33.3 \%$ & 0.85 \\
Adenomatous polyps & 3 & $17.7 \%$ & 21 & $22.6 \%$ & 0.65 \\
Multiple adenomatous polyps & 1 & $5.9 \%$ & 7 & $7.5 \%$ & 0.81 \\
Low grade dysplasia & 3 & $17.6 \%$ & 18 & $19.3 \%$ & 0.85 \\
High grade dysplasia & 1 & $5.9 \%$ & 3 & $3.2 \%$ & 0.59 \\
\hline
\end{tabular}

Data are number of individuals (\%); CRC, colorectal cancer

might be an accelerated adenomas formation, as seen in patients with HNPCC. However, two prospective studies have been published recently $[25,26]$. They reported no increased risk among IPMN patients for all incident extrapancreatic malignancies, including CRC. As these are so far the largest available prospective data on IPMN and CRC, it could be that the reason we could not detect any increased risk for adenomatous polyps in IPMN patients depicts a lack of significant association between IPMN and CRC.

We observed several risk factors associated with IPMN. History of diabetes, use of insulin and history of chronic pancreatitis has been previously reported to be associated with IPMN [20]. We found an association between cholecystectomy and IPMN, which has never been reported before and the meaning of which appears unclear.

We acknowledge that our study has some limitations. It included a relatively limited number of IPMN cases, and because of the small number of CRCs detected, it was unable to detect a difference in the CRC occurrence as compared to matched controls. However, the study was designed to assess the prevalence of adenomas and not of CRC in the IPMN group. Furthermore, a significantly higher rate of chronic pancreatitis and heavy drinking in the IPMN group suggests misclassification indicating that some of the cystic lesions may be pseudocysts rather than IPMNs. However, this is the first study assessing the prevalence of colorectal adenomas in IPMN patients at a first time colonoscopy. Although the results reported were against our prior hypothesis of a higher prevalence of colorectal adenomas among IPMN patients than in the control population, our study highlighted the need to further explore the issue of association between IPMN and CRC.

\section{CONCLUSION}

Patients wiht IPMN are not at an increased risk for development of adenomatous colorectal polyps. Further research is required in order to assess if there is actually a real association between IPMN and CRC.

Conflicts of interest: None declared.
Authors' contribution: G.C., A.L., G.D.F. and G.C.S. (Guido Costamagna) designed the study. G.V. and S.S. collected the data and interviewed the patients. F.A., A.L. and E.D.G. conducted colonoscopies. E.L. conducted statistical analysis. N.P. drafted the manuscript. G.C., A.L., S.B. and M.B. revised the manuscript.

Acknowledgement: The work of N.P. was supported by the ERAWEB project, funded with the support of the European Community.

\section{REFERENCES}

1. Sugiyama M, Atomi Y. Extrapancreatic neoplasms occur with unusual frequency in patients with intraductal papillary mucinous tumors of the pancreas. Am J Gastroenterol 1999; 94: 470-473. doi: 10.1111/j.15720241.1999.879_h.x

2. Choi MG, Kim SW, Han SS, Jang JY, Park YH. High incidence of extrapancreatic neoplasms in patients with intraductal papillary mucinous neoplasms. Arch Surg 2006; 141: 51-56. doi: 10.1001/ archsurg.141.1.51

3. Eguchi H, Ishikawa O, Ohigashi $\mathrm{H}$, et al. Patients with pancreatic intraductal papillary mucinous neoplasms are at high risk of colorectal cancer development. Surgery 2006; 139:749-754. doi: 10.1016/j. surg.2005.11.008

4. Oh SJ, Lee SJ, Lee HY, et al. Extrapancreatic tumors in intraductal papillary mucinous neoplasm of the pancreas. Korean J Gastroenterol 2009; 54: 162-166. doi: 10.4166/kjg.2009.54.3.162

5. Yoon WJ, Ryu JK, Lee JK, et al. Extrapancreatic malignancies in patients with intraductal papillary mucinous neoplasm of the pancreas: prevalence, associated factors, and comparison with patients with other pancreatic cystic neoplasms. Ann Surg Oncol 2008; 15: 3193-3198. doi: 10.1245/s10434-008-0143-4

6. Riall TS, Stager VM, Nealon WH, et al. Incidence of additional primary cancers in patients with invasive intraductal papillary mucinous neoplasms and sporadic pancreatic adenocarcinomas. J Am Coll Surg 2007; 204: 803-813. doi: 10.1016/j.jamcollsurg.2007.01.015

7. Kamisawa T, Tu Y, Egawa N, Nakajima H, Tsuruta K, Okamoto A. Malignancies associated with intraductal papillary mucinous neoplasm of the pancreas. World J Gastroenterol 2005; 11: 5688-5690. doi: 10.3748/wjg.v11.i36.5688 
8. Reid-Lombardo KM, Mathis KL, Wood CM, Harmsen WS, Sarr MG. Frequency of extrapancreatic neoplasms in intraductal papillary mucinous neoplasm of the pancreas: implications for management. Ann Surg 2010; 251: 64-69. doi: 10.1097/SLA.0b013e3181b5adle

9. Baumgaertner I, Corcos O, Couvelard A, et al. Prevalence of extrapancreatic cancers in patients with histologically proven intraductal papillary mucinous neoplasms of the pancreas: a case-control study. Am J Gastroenterol 2008; 103: 2878-2882. doi: 10.1111/j.1572-0241.2008.02142.x

10. Ishida M, Egawa S, Kawaguchi K, et al. Synchronous and metachronous extrapancreatic malignant neoplasms in patients with intraductal papillary-mucinous neoplasm of the pancreas. Pancreatology 2008; 8: 577-582. doi: 10.1159/000159844

11. Lubezky N, Ben-Haim M, Lahat G, et al. Intraductal papillary mucinous neoplasm of the pancreas: associated cancers, family history, genetic predisposition? Surgery 2012; 151: 70-75. doi: 10.1016/j.surg.2011.06.036

12. Kawakubo $\mathrm{K}$, Tada $\mathrm{M}$, Isayama $\mathrm{H}$, et al. Incidence of extrapancreatic malignancies in patients with intraductal papillary mucinous neoplasms of the pancreas. Gut 2011; 60: 1249-1253. doi: 10.1136/gut.2010.227306

13. Larghi A, Panic N, Capurso G, et al. Prevalence and risk factors of extrapancreatic malignancies in a large cohort of patients with intraductal papillary mucinous neoplasm (IPMN) of the pancreas. Ann Oncol 2013; 24: 1907-1911. doi: 10.1093/annonc/mdt184

14. Ferlay J SI, Ervik M, Dikshit R, et al. GLOBOCAN 2012 v1.0, Cancer Incidence and Mortality Worldwide: IARC CancerBase No. 11. 2013 10.1002/ijc.26192; Available from: http://globocan.iarc.fr

15. Siegel R, DeSantis C, Virgo K, et al. Cancer treatment and survivorship statistics, 2012. CA Cancer J Clin 2012; 62: 220-241. doi: 10.3322/ caac. 21149

16. Brenner H, Bouvier AM, Foschi R, et al. Progress in colorectal cancer survival in Europe from the late 1980s to the early 21st century: the EUROCARE study. Int J Cancer 2012; 131: 1649-1658. doi: 10.1002/ ijc. 26192
17. Center MM, Jemal A, Smith RA, Ward E. Worldwide variations in colorectal cancer. CA Cancer J Clin 2009; 59: 366-378. doi: 10.3322/ caac. 20038

18. Sankaranarayanan R, Swaminathan R, Brenner H, et al. Cancer survival in Africa, Asia, and Central America: a population-based study. Lancet Oncol 2010; 11: 165-173. doi: 10.1016/S1470-2045(09)70335-3

19. Jass JR. Classification of colorectal cancer based on correlation of clinical, morphological and molecular features. Histopathology 2007; 50: 113-130. doi: 10.1111/j.1365-2559.2006.02549.x

20. Capurso G, Boccia S, Salvia R, et al. Risk factors for intraductal papillary mucinous neoplasm (IPMN) of the pancreas: a multicentre casecontrol study. Am J Gastroenterol 2013; 108: 1003-1009. doi: 10.1038/ ajg. 2013.42

21. Levy P, Jouannaud V, O'Toole D, et al. Natural history of intraductal papillary mucinous tumors of the pancreas: actuarial risk of malignancy. Clin Gastroenterol Hepatol 2006; 4: 460-468. doi: 10.1016/j. cgh.2006.01.018

22. Lieberman DA; American Gastroenterological Association. Colon polyp surveillance: clinical decision tool. Gastroenterology 2014; 146: 305-306. doi: 10.1053/j.gastro.2013.11.029

23. Kinzler KW, Vogelstein B. Lessons from hereditary colorectal cancer. Cell 1996; 87: 159-170. doi: 10.1016/S0092-8674(00)81333-1

24. Fearon ER. Molecular genetics of colorectal cancer. Annu Rev Pathol 2011; 6: 479-507. doi: 10.1146/annurev-pathol-011110-130235

25. Marchegiani G, Malleo G, D’Haese JG, et al. Association between pancreatic intraductal papillary mucinous neoplasms and extrapancreatic malignancies. Clin Gastroenterol Hepatol 2015; 13: 1162-1169. doi: 10.1016/j.cgh.2014.11.029

26. Malleo G, Marchegiani G, Borin A, et al. Observational study of the incidence of pancreatic and extrapancreatic malignancies during surveillance of patients with branch-duct intraductal papillary mucinous neoplasm. Ann Surg 2015; 261: 984-990. doi: 10.1097/ sla.0000000000000884 